\title{
Biochemistry of Human Axilla Malodor and Chemistry of Deodorant Ingredients
}

\author{
Markus Gautschi*, Andreas Natsch, and Fridtjof Schröder
}

\begin{abstract}
Key human axilla malodorants are hexenoic acid (3) and 3-hydroxy-3-methyl-hexanoic acid (5), accompanied by some 25 structurally related hydroxyalkanoic acids and alkenoic acids. These sweat acids are secreted in the axilla in the form of odorless glutamine conjugates and are released upon enzymolysis by AMRE (Axillary Malodor Releasing Enzyme), produced by Corynebacteria. The sulfanylalkanols 8-11 represent another important group of axilla malodor compounds that are also secreted in the form of odorless precursors. The major precursors are cysteine-glycine sulfanylalkanol conjugates of type 14 and the minor precursors are cysteine sulfanylalkanol conjugates like 15. The release occurs upon action of a $\beta$-lyase of axilla bacteria. Besides the classical approaches of axilla malodor masking using fragrances, the use of chemicals to neutralize malodorants is described. The elucidation of the biochemistry of the sweat acid release has allowed the development of fragrance precursors that act as competitive substrates to the natural malodor precursors as well as the development of specific antagonists that block AMRE. Finally, the characterization and functional expression of a first human malodor receptor presents an interesting approach for future development of axilla malodor blockers.
\end{abstract}

Keywords: Axillary body malodorant - Axillary malodor releasing enzyme - Fragrance precursor . Human body malodor · Inhibitor - Malodor counteraction · Malodor precursor

\section{Introduction}

Human body odors are perceived by the modern society as offensive. Therefore, over the past years significant efforts have been made to eliminate or mask body odors and the deodorant and antiperspirant sector has become one of the major segments of the cosmetic industry. The strongest body odors generally originate from the axillary region. This particular region of the skin harbors a dense array of different glands, with the so-called apocrine glands being the key source of body odors. Since the original work of Shelley et al. in the early fifties [1], it is known that sweat secreted by the apocrine glands is odorless and the typical and familiar sweat smell only develops upon bacterial action. From these early observations it could be concluded that

\footnotetext{
${ }^{\star}$ Correspondence: Dr. M. Gautschi

Givaudan Schweiz AG

Fragrance Research

Ueberlandstrasse 138

$\mathrm{CH}-8600$ Dübendorf

Tel.: +41448242324

Fax: + 41448242926

E-Mail: markus.gautschi@givaudan.com
}

sweat contains precursors for odorants - or better malodorants - that are released due to enzymatic action of bacterial enzymes. It has taken 50 years since these initial observations to elucidate the key molecules and enzymes that are involved in this process. The goal of this article is to give an overview of these recent findings. Furthermore, we present classical approaches to reduce or counteract human body malodor and describe how the understanding of the malodor formation at the molecular level is being used in the development of novel deodorant ingredients.

\section{Malodor Formation}

\subsection{Chemical Nature of Body Malodorants}

In early analytical studies, the steroids 5 $\alpha$-androst-16-en-3-one (1) [2], which had previously been known as a boar pheromone, and $5 \alpha$-androst-16-en-3 $\alpha$-ol (2) [3] had been detected and proposed as principal odoriferous contributors in human axilla secretions (Fig. 1). However, this did not correspond with how these two components are perceived. For example the smell of androstenone $\mathbf{1}$ is described as urine-like and offensive, and its overall contribution to the axillary malodor is therefore probably not relevant, even more so as $\mathbf{1}$ is only perceived by $50 \%$ of the human population. Several new malodor components were identified by the detailed analysis of the chemical composition of axillary odor carried out by Zeng et al. [4]. They concluded that (E)-3methyl-2-hexenoic acid (3) is the key odor component in sweat, accompanied by several other carboxylic acids such as 4-ethyloctanoic acid (4), which is well known by flavorists and is nicknamed 'goat-acid' due to its typical odor. The hexenoic acid $\mathbf{3}$ had originally been only isolated from the skin of people suffering from schizophrenia, and Zeng et al. were the first to show its occurrence in the general population.

We started investigating human axilla malodor in the early nineties and we confirmed the presence of $\mathbf{3}$ in significant amounts in hydrolyzed axillary secretions. Additionally, 3-hydroxy-3-methyl-hexanoic acid (5), the hydrated analogue of 3 , could be identified for the first time. Quantitatively, $\mathbf{5}$ is the most abundant component and its inherent odor described as very pungent, sweat-like [5], together with an extremely low odor threshold value of $0.0044 \mathrm{ng} / \mathrm{l}$, underlines the importance of $\mathbf{5}$ for the overall hedonic character of sweat. Several years later, when we had obtained a small amount of the metallopeptidase described below, we could identify - following enzymolysis of sweat samples and subsequent in-depth analyses - some 25 novel hydroxyalkanoic 


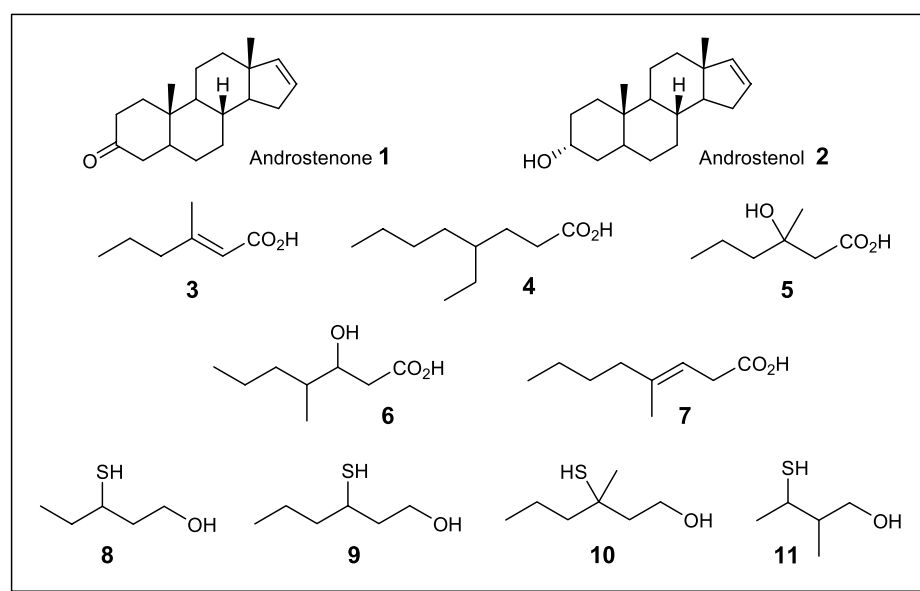

Fig. 1. Key impact body malodorants

acids and alkenoic acids related to $\mathbf{3}$ and $\mathbf{5}$. These acids, such as the two typical examples 6 and 7 (Fig. 1), had similar pungent sweat-like odor, although less pronounced [6]. These acids were found in much lower quantities but the fact that the interpersonal variability was much higher than for $\mathbf{3}$ and $\mathbf{5}$ may lead to the conclusion that they are key determinants of 'body odor individuality'.

Besides these acids, a second class of compounds plays a key role in the perception of sweat malodor. The sulfanylalkanols 8-11 were identified independently by two research groups as impact constituents of sweat extracts [7][8]. Although present in trace quantities only, these sulfanylalkanols contribute to the overall body malodor, which is due to their extraordinarily low odor threshold values in the range of $1 \mathrm{pg} / \mathrm{l}$ [7] and their high volatility. The sulfanylalkanol 10, which was reported at the same time by a third research group [9], is quantitatively the most dominant component amongst all identified sulfanylalkanols. Its structural similarity to the two dominant malodorant acids $\mathbf{3}$ and $\mathbf{5}$ is striking and one can assume a common biosynthetic origin.

\subsection{Structure of the Natural Precursors for Body Odorants}

Many years following the initial work of Shelley et al. [1], only speculations with respect to the chemical nature of the sweat malodorant precursors were available. It was proposed that androstenol $\mathbf{2}$ is released by bacterial enzymes from the corresponding sulfate and glucuronide that are secreted into the axilla [10]. However, neither could the steroid precursors be isolated from axilla secretions, nor was there clear evidence for enzymes in skin bacteria able to cleave sulfate- and glucuronide-conjugates of steroids. For the release of sulfanylalkanols the putative implication of a pyridoxal-phosphate dependent $\beta$-lyase has been proposed [11], but no data related to the structure of the secreted precursor(s) or the nature of

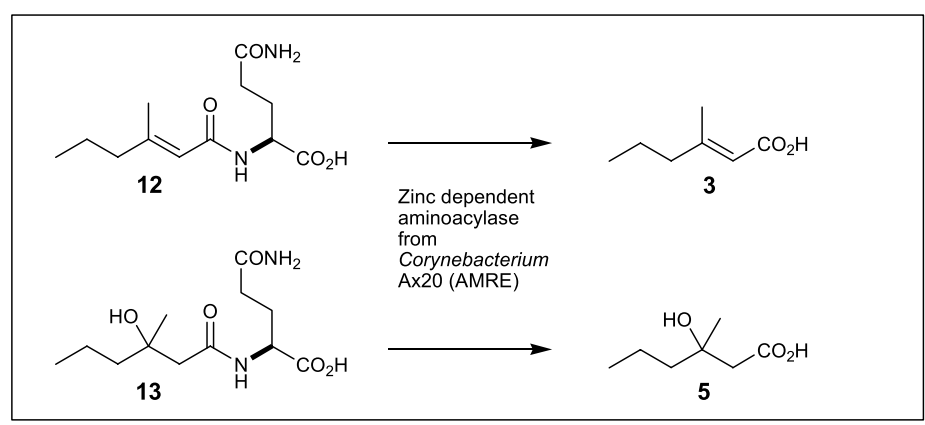

Scheme 1. Release of sweat acids from glutamine

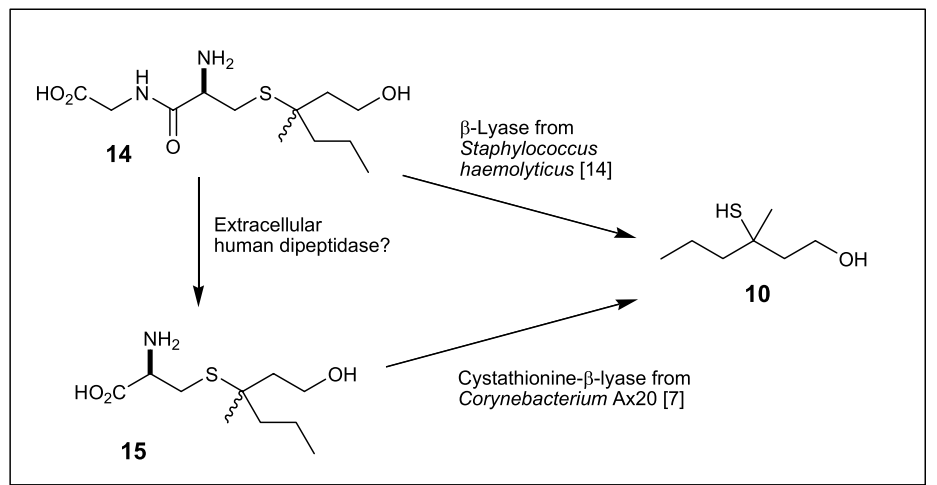

Scheme 2. Release of malodorant sulfanylalkanol 10 from precursors 14 and 15

the bacterial enzyme was given. Zeng et al. were the first to investigate the chemical nature of the precursors for the malodorant acids and showed that $\mathbf{3}$ is released upon incubation of the aqueous fraction of apocrine secretions with Corynebacteria or upon hydrolysis with $\mathrm{NaOH}$ [12]. This was a first indication that the acids must be covalently bound to a water-soluble carrier moiety. The same research group reported a few years later that $\mathbf{3}$ is non-covalently associated with apolipoprotein D [13], the major protein present in axilla secretions. In contrast to their earlier work, they now proposed this apolipoprotein-acid complex to be the malodorant acid precursor present in axilla sweat.

Firm analytical evidence for the nature of malodorant precursors, both for the acids and the sulfanylalkanols, has only been found recently. We reported for the first time the presence of the glutamine conjugates 12 and 13 (Scheme 1) in fresh axilla secretions [5], a finding that was confirmed later on [14]. In subsequent studies we also found analytical evidence for the presence of the corresponding glutamine conjugates of the acids 6 and 7 [6], two compounds that have been identified in sweat samples that were treated with the glutamine-specific metallopeptidase reported in Section 2.3. The occurrence of the glutamine derivatives $\mathbf{1 2}$ and $\mathbf{1 3}$ as well as the corresponding glutamine derivatives of the acids $\mathbf{6}$ and $\mathbf{7}$ and the fact that all the different acids are released from sweat by the glutamine-specific metallopeptidase strongly supports the assumption that all sweat acids share a common glutaminebased precursor principle.

The structure elucidation of the precursor for the sulfanylalkanols has proven somewhat more complex and some open questions still need to be answered. Initially we reported that upon treatment of the cysteine derivative $\mathbf{1 5}$ (Scheme 2) with a $\beta$-lyase cloned from Corynebacterium Ax20, the sulfanylalkanol 10 is released [7]. Similarly, this $\beta$-lyase does release $\mathbf{1 0}$ from axilla secretions, indicating that $\mathbf{1 5}$ indeed is a precursor present in axilla secretions. However, Starkenmann et al. have shown strong evidence that in fact the dipeptide conjugate $\mathbf{1 4}$ is the precursor for the sulfanylalkanol 10 (Scheme 2) [14]. The CysGly compound $\mathbf{1 4}$ is most likely derived from a glutathione adduct, but this adduct itself does not appear to be secreted [14].

\subsection{Biochemistry of Odorant Release from the Precursors}

Following the isolation of the acid precursors 12 and 13, it has been shown that these glutamine derivatives are cleaved by bacterial strains isolated from the skin in the axillary regions [5]. Interestingly, the precursors are only cleaved by bacteria belonging to the genus Corynebacteria, which is in accordance with earlier observations that only human subjects with a large population of Corynebacteria in the axilla have significant body odor formation [16]. In contrast, isolates of Staphylococcus epidermidis, which are also abundant inhabitants of the axillary regions, are unable to cleave these substrates. Subsequently, abacterial enzyme 
cleaving 12 and 13 was isolated by classical biochemical purification techniques from a strain of Corynebacterium striatum [5]. This enzyme was found to be inhibited by $o$-phenanthrolin, but not by other protease inhibitors targeting serine-, cysteine- or aspartate-proteases, indicating that it belongs to the class of metallopeptidases. The gene coding for this enzyme could be cloned [5] and a close phylogenetical relationship with other zinc-dependent metallopeptidases has been established. Following the heterogeneous expression of this enzyme in E. coli, it has been shown that this single recombinant enzyme can not only release the key sweat acids 3 and 5 (Scheme 1), but also releases a very broad range of odorant acids such as 4, 6 and 7 from axilla secretions [7], as already described above. These findings further demonstrated the importance of this enzyme in body malodor formation and therefore it was named AMRE - Axillary Malodor Releasing Enzyme.

In order to investigate the release of the sulfanylalkanols from sweat, the gene coding for a cystathionine- $\beta$-lyase was isolated from the same bacterial strain Ax20 that served as a source for the metallopeptidase AMRE. This enzyme has been expressed in $E$. coli and shown to release the sulfanylalkanol 10 from odorless axilla secretions and it did cleave the pure compound 15 (Scheme 2), supporting its involvement in axilla odor formation [6]. The dipeptide derivative $\mathbf{1 4}$ has been shown to release $\mathbf{1 0}$ upon the action of Staphylococcus haemolyticus [14], which stands in some contrast to the general observation that body odorants are mainly released by Corynebacteria. However, the enzyme mediating this cleavage has not yet been reported. Prior to the action of the corynebacterial $\beta$-lyase, compound 14 could also be transformed into 15 by an eukaryotic extracellular dipeptidase which is known to be involved in the metabolism of glutathione conjugates [17], but a bacterial transformation cannot be ruled out either.

\section{Approaches to Counteract Body Malodor}

The first trademarked underarm deodorant was introduced in the United States in 1888 under the brand name of Mum [18]. It was a waxy cream and probably controlled odor by virtue of the weak antibacterial action of zinc oxide it contained. More than hundred years later, a deodorant under the Mum brand name is still on the market, but in addition numerous other antiperspirants and deodorants are available and come in many forms such as creams, powders, aerosols, pump sprays, roll-ons etc. Although the terms antiperspirant and deodorant are often used interchangeably, they represent two different and distinct actions. Antiperspirant specifically refers to materials or formulations that reduce the underarm perspiration, whereas deodorant refers to materials or formulations which mask or inhibit the formation of unpleasant body odors [18].

In the following we will describe existing approaches to counteract body malodor and describe some of the deodorant ingredients that have been or are still being used, without being comprehensive. The new insights into the biochemistry of body malodor formation described in the preceding Section and summarized in Fig. 2, opened up new concepts to fight body malodor that are currently being explored. First results of these activities towards the development of potential, new deodorant ingredients are presented.

\subsection{Body Malodor Modification - Deodorant Fragrances}

The most traditional method of achieving deodorancy is the use of fragrances to mask body malodor. This may involve simple overpowering of the malodor with a pleasant fragrance or a more sophisticated approach in which the fragrance is designed such that it harmonizes well with the malodor so that overall the perceived character is more pleasant. This involves on one hand the knowledge of compounds that harmonize well with the sweat malodor components and, on the other hand, the art of perfumery. Such ingredients that do not possess themselves an intense odor but have malodor harmonizing and/or counteracting effects have long been used, e.g. the compounds formerly sold under the trade name of Veilex [19] (Fig. 3), and the search for new compounds having these properties is still ongoing [20].

Malodor masking with perfumes is usually achieved in combination with ingredients capable of chemically removing body malodorants to an extent that masking is more effective. The deodorant activity of antiperspirant salts is attributed to the chemical malodor-removal effect, and several metal salts such as zinc carbonate, magnesium or lanthanum oxides, hydroxides or carbonates, or mixtures thereof, have been claimed to have such effects [21]. Especially zinc salts have been used for their malodor neutralizing effects, examples being zinc glycinate [22] and zinc ricinoleate, a key active ingredient in commercial products such as Grillocin ${ }^{\mathrm{TM}}$ [23] and Tego ${ }^{\circledR}$ Sorb [24]. The action of these salts is explained by interaction of the zinc cation with the malodorants, most likely the sulfanylalkanols 8-11, in an aqueous environment [25], which ultimately facilitates the masking of the reduced malodor by the fragrance.

Other compounds that have been used are those which can react with the malodor components, for example materials that have a carbon-carbon double bond conjugated with one or more carbonyl groups. Aldehydes are frequently used materials of this class to counteract malodor, the most commonly used ones for deodorant proper-

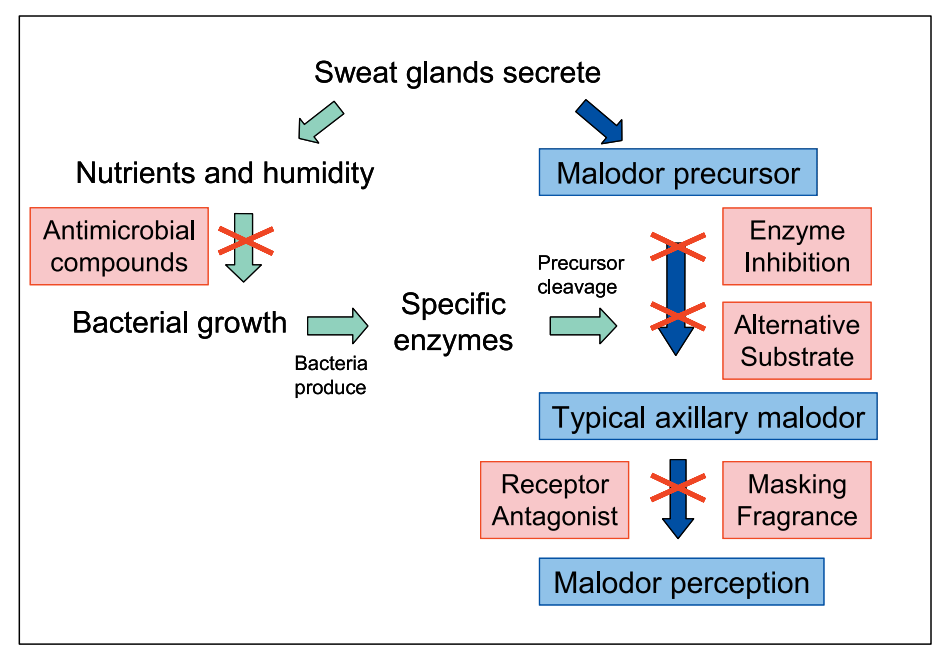

Fig. 2. Intervention strategies for deodorant ingredients

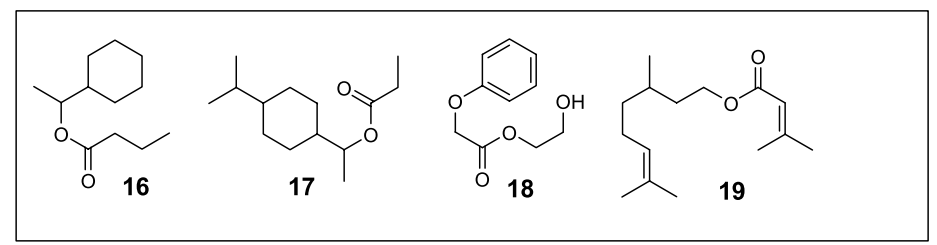

Fig. 3. Selection of compounds $\mathbf{1 6 - 1 8}$ formerly sold under the name of Veilex $^{\mathrm{TM}}$, and citronellyl senecioate 19 
ties are trimethylhexanal, other alkyl-substituted aldehydes, benzaldehyde, and vanillin [26]. Esters of alpha-, beta-unsaturated monocarboxylic acids as well as aromatic unsaturated carboxylic acid esters wherein the unsaturation is conjugated to both the aromatic ring and the ester carbonyl group, have been found to be useful as malodor counteractants [27]. Commercial products from this type of compounds include e.g. citronellyl senecioate 19 - Sinodor ${ }^{\mathrm{TM}}$ [28]. Recently also cyclodextrins have been claimed to be effective in body odor reduction [29].

\subsection{Antibacterial Compounds}

The connection of body malodor and axillary bacterial action was already established in the late 1940s and ever since antibacterial agents have been widely used in deodorants and antiperspirants. In the fifties and sixties, hexachlorophene $\mathbf{2 0}$ was the antimicrobial compound most widely used in deodorants and deodorant soaps, but also many other halogenated compounds were introduced. In the early seventies, hexachlorophene $\mathbf{2 0}$ was banned due to toxicological concerns and at that time triclosan 21, and to a lesser extent triclocarban 22 (Fig. 4), became the key antimicrobial compounds in the cosmetic industry, finding a broad use in all kinds of deodorant products [30]. The use of the polychlorinated triclosan 21 in cosmetic products has repeatedly earned critics from consumer organizations and several deodorant manufacturers have abandoned its use over time. For several years the natural sesquiterpene farnesol 23, which has a potent bacteriostatic action against skin bacteria, was used as an alternative [31]. However, occasional skin sensitization reactions to this compound were recorded and its use is now more and more reduced. Another alternative introduced is DTPA [32]. This compound has a strong chelating activity for iron, thus reducing the availability of iron in the axilla, which can be a growth limiting factor for bacteria. Other compounds occasionally used include antibacterial quaternary ammonium compounds and biguanides. Since toxicological concerns were often the reason for the reduced use of certain antimicrobials, also rather weak antibacterial compounds have been introduced to the market in recent years. This is illustrated with the glycol ether 25 (Fig. 4), an example of a mild antibacterial compound used in some deodorant brands [33].

\subsection{AMRE-Precursors and -Inhibitors}

Eliminating the natural skin microflora with antibacterial chemicals is a relatively crude approach for malodor control and the trend in the cosmetic industry for mild and minimally invasive products calls for alter-

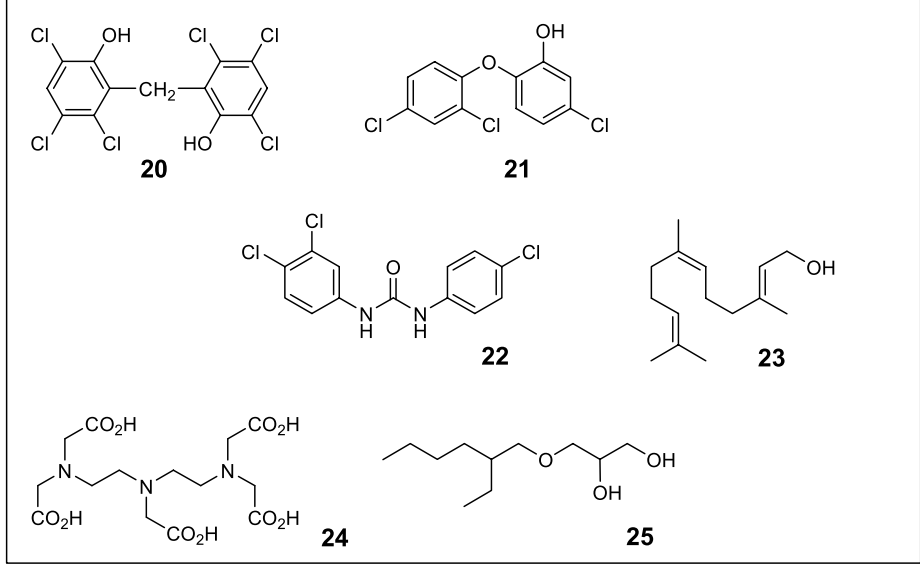

Fig. 4. Some classical and new antibacterial deodorant ingredients

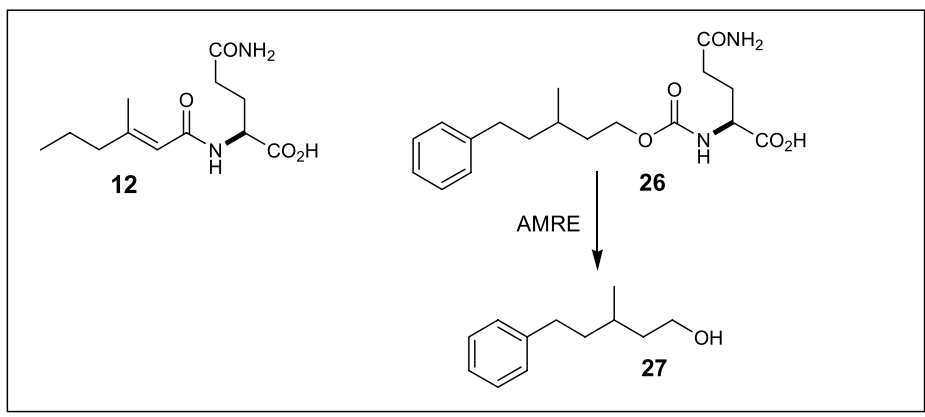

Scheme 3: Release of phenoxanol 27 from precursor 26 [34], in analogy to the release of sweat acid 3 from natural precursor 12 (Scheme 1)

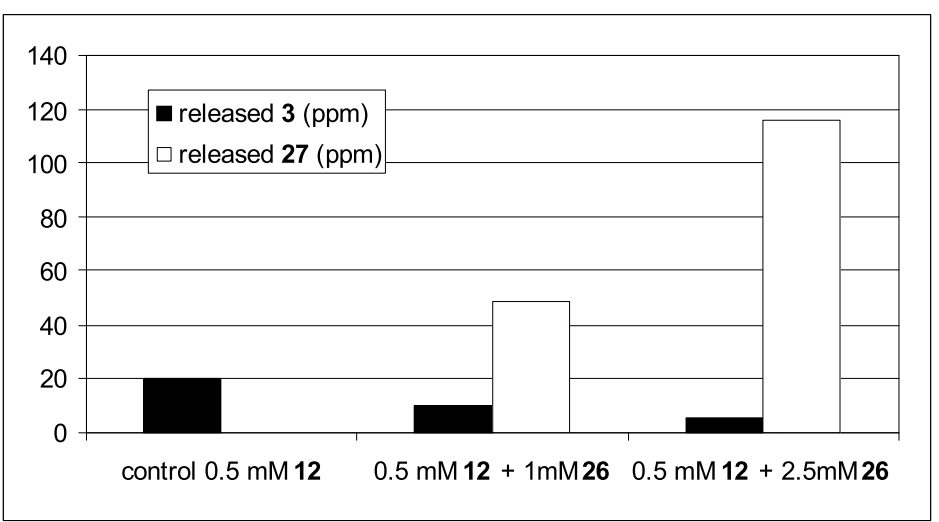

Fig. 5. Inhibition of $\mathbf{1 2}$ by competitive substrate 26

natives. The knowledge of the specificity of the biochemical process of malodor formation - outlined in Section 2.3 and illustrated in Fig. 2 - allowed us to investigate a more elegant approach to control malodor formation, targeting AMRE with either enzyme inhibitors or alternative substrates. AMRE has a unique substrate specificity: on one hand only $\mathrm{N}_{\alpha}$-substituted glutamine-conjugates and no derivatives of other amino acids, not even of closely related ones, are recognized, whereas a large range of different hydrophobic $\mathrm{N}_{\alpha}$-substituents are tolerated [5]. Consequently we first explored the possibility of replacing the malodorant acids by a fragrant ingredient. We have found that carbamates such as compound $\mathbf{2 6}$ are indeed recognized by AMRE (Scheme 3)
[34] and a broad range of fragrance alcohols can be efficiently released [35].

These compounds use the natural precursor principle and leave the natural skin microflora intact as they do not kill bacteria unlike compounds such as triclosan 21. Yet triclosan has been outperformed in clinical studies by these new fragrance precursors [36], making them very attractive to be used as new deodorant ingredients. Using in vitro tests, we could show that by increasing the concentration of the fragrance precursor 26 in presence of a constant amount of malodor precursor $\mathbf{1 2}$ a dual benefit is achieved: the amount of released fragrance alcohol 27 increases whereas the release of the sweat acid 3 is significantly reduced (Fig. 5) [34]. The disadvantage of this competitive mode 
of action is the fact that these fragrance precursors need to be present in excess over the natural substrate in order to be efficient.

The disadvantages of such competitive substrates can be overcome by the use of antagonists that block the enzyme. The development of specific and stable enzyme inhibitors active at much lower concentrations was therefore the next major challenge. Based on the fact that AMRE is a zinc peptidase and based on the detailed knowledge of the substrate specificity, new enzyme inhibitors were designed by replacing the cleavable carbamate unit of $\mathbf{2 6}$ by another non-cleavable functional group. The phosphine group is one of the functional groups often used by medicinal chemists when designing peptidase inhibitors [37]. Indeed, the first substituted phosphine compounds of type $28(\mathrm{R}=\mathrm{Pr}$, $\mathrm{Pr}, \mathrm{Ph}$ see Fig. 6 ), were found to be promising antagonists of AMRE, with $\mathrm{IC}_{50}$ values ranging from 180 to $440 \mathrm{nmol}$ [38].

In a first lead optimization step we explored modifications at the glutamine backbone of the precursor. However, any substitution of glutamine resulted in complete loss of activity $\left(\mathrm{IC}_{50}>40000 \mathrm{nmol}\right)$ of the precursors, which confirmed the very high glutamine specificity of AMRE. In the next optimization step we explored the scope and limits of the substituent R of the phosphine group. From our work with the precursors $\mathbf{2 6}$ we expected a fairly broad tolerance, hence the task was to identify the optimal substituent R. After screening of a large number of inhibitors we had identified compounds like 29 and $\mathbf{3 0}$ (Fig. 6) with excellent $\mathrm{IC}_{50}$ values of 19 and $11 \mathrm{nmol}$, respectively [39], which are by a factor of 20-40 better than the one of the original lead compound $\mathbf{2 8}$ ( $\mathrm{R}=$ propyl). Currently, these compounds are further optimized in terms of activity, synthesis and bioavailability.

\subsection{Olfactory Receptor Antagonists}

Already in the early 80 s, the deodorant properties of some plant extracts were attributed to a complex mechanism, including the inhibition of nasal olfactory receptors [40]. The pioneering work of Buck and Axel [41], the identification of a novel multigene family that encodes for odorant receptors, laid the basis for the understanding of odor perception at the receptor level. The first step involves the detection of odorants, and hence also malodorants, by $\mathrm{G}$ protein-coupled odorant receptors on olfactory sensory neurons (OSNs) in the nasal olfactory epithelium [42]. In response to odorants these OSNs transmit signals to the brain, thereby generating the odor perceptions. Each OSN expresses a single functional odorant receptor gene [43], and OSNs with the same odorant receptor are randomly dispersed within one olfactory

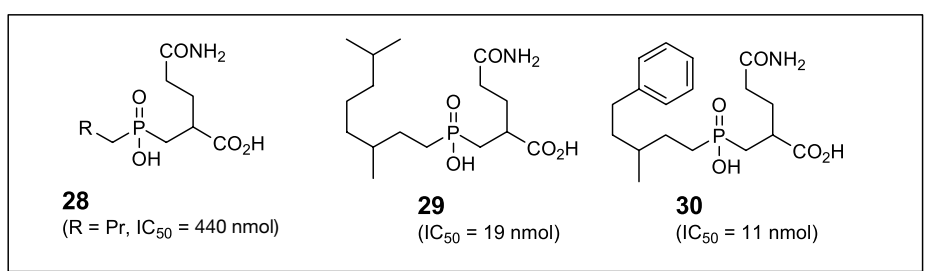

Fig. 6. First and second generation AMRE inhibitors

epithelial zone [44]. Mammals have as many as 1000 different odorant receptors that are used combinatorially to detect and discriminate diverse smelling odorants and malodorants [43]. Recent efforts in this field have not only led to the identification of $c a .380$ functional human odorant receptors [45], but finally, after years of research, also cell systems are available that allow the functional expression of these receptors [46][47]. A few months ago the identification of the olfactory receptor RCC356 responding to the malodorant isovaleric acid was reported [48]. Functional expression of this receptor will help to identify compounds that can act as malodor blockers at the receptor level. Similarly, receptors responding to body malodorants will be used as biosensors to identify antagonists.

\section{Conclusion and Outlook}

Fundamental research on body malodorants, their precursors, and the enzymes cleaving them has led to a detailed understanding of the biochemical processes. Microbial formation of axilla malodor is not, like in other cases of microbial malodor formation, an unspecific putrefaction process in which degradation of lipids and proteins yields common simple odoriferous metabolites, but involves a very specific transformation of secreted metabolites which appear to be specific to the human physiology. This has both a scientific and a practical implication: on the scientific side, it indicates that the axillary organ [49] is a scent gland producing specific metabolites which, at least in evolutionary history of man, must have had some signal function. On the practical side, the specificity of these processes opened up new ways for targeted chemical malodor counteraction, e.g. with glutamine-derived fragrance precursors and with AMRE inhibitors that show promising deodorant properties. Finally, functional expression of human olfactory receptors responding to body malodorants may lead to the search for effective antagonists.

Received: October 28, 2006

[1] W.B. Shelley, H.J. Hurley, A.C. Nichols, Arch. Derm. Syphilol. 1953, 68, 430.

[2] R. Claus, W. Alsing, J. Endocrinol. 1976, 68, 483.
[3] B.W.L. Brooksbank, R. Brown, J.A. Gustafsson, Experientia 1974, 30, 864.

[4] X.N. Zeng, J.J. Leyden, H.J. Lawley, K. Sawano, I. Nohara, G. Preti, J. Chem. Ecol. 1991, 17, 1469.

[5] A. Natsch, H. Gfeller, P. Gygax, J. Schmid, G. Acuña, J. Biol. Chem. 2003, 278, 5718.

[6] A. Natsch, S. Derrer, F. Flachsmann, J. Schmid, Chemistry \& Biodiversity 2006, 3,1 .

[7] A. Natsch, J. Schmid, F. Flachsmann, Chemistry \& Biodiversity 2004, 1, 1058.

[8] a) Y. Hasegawa, M. Yabuki, M. Matsukane, Chemistry \& Biodiversity 2004, 1 , 2042; b) M. Yabuki, Y. Hasegawa, M. Matsukane, in 'International Symposium on the Chemistry of Essential Oils, Terpenes and Aromatics, Tokushima Bunri University, October 18-20, 2002, Tokushima, Japan, p. 124; c) Y. Hasegawa, M. Furukawa, M. Matsukane, JP 10025265, priority 12.7.1996 to Kao Corp., Chem. Abstr. 128, 127752.

[9] M. Troccaz, C. Starkenmann, Y. Niclass, M. van de Waal, A.J. Clark, Chemistry \& Biodiversity 2004, 1, 1022.

[10] C. Froebe, A. Simone, A. Charig, E. Eigen, J. Soc. Cosmet. Chem. 1990, 41, 173.

[11] S.B. Lyon, C. O'Neal, H. Van der Lee, B. Rogers, WO 9105541, priority 10.10 .1989 to Gillette Co., Chem. Abstr. 115, 99001.

[12] X.N. Zeng, J.J. Leyden, J.G. Brand, A.I Spielman, K.J. McGinley, G. Preti, J. Chem. Ecol. 1992, 18, 1039.

[13] C. Zeng, A.I. Spielman, B.R. Vowels, J.J. Leyden, K. Biemann, G. Preti, Proc. Natl. Acad. Sci. USA 1996, 93, 6626.

[14] C. Starkenmann, Y. Niclass, M. Troccaz, A.J. Clark, Chemistry \& Biodiversity 2005, 2, 705.

[15] J.J. Leyden, K.J. McGinley, E. Hoelzle, J.N. Labows, A.M. Klingman, J. Invest. Dermatol. 1981, 77, 413.

[16] a) J.J. Leyden, K.J. McGinley, E. Hoelzle, J.N. Labows, A.M. Klingman, J. Invest. Dermatol. 1981, 77, 413; b) P.J. Jackman, W.C. Noble, Clin. Exp. Dermatol. 1983, 8 , 259.

[17] A. Meister, Methods in Enzymology 1995, 251,3 .

[18] K. Laden, 'Introduction and History of Antiperspirants and Deodorants' in 'Antiperspirants and Deodorants', Ed. K. Laden, C.B. Felger, Marcel Dekker Inc., New York, 1988, p 1.

[19] a) A. Schleppnik, DE 2650602, priority 11.4.1976 to Monsanto Co., Chem. Abstr. 
87, 73244; b) A. Schleppnik, S.G. Vanata, US 4009253, priority 17.3.1975 to Monsanto Co., Chem. Abstr. 86, 177184.

[20] U. Rohde, S. Hillers, H. Surburg, S. Sonnenberg, K. McDermott, L. Smith, K. Sparkuhle, WO 2001043784, priority 12.8.2000 to Haarmann \& Reimer G.m.b.h., Chem. Abstr. 135, 66066.

[21] B. Bews, P. Critchley, J.A. Durrant, M.R.D. Stebles, L.R.H. Tipping, EP 24176, priority 8.8.1980 to Unilever Ltd., Chem. Abstr. $95,12585$.

[22] F.W. Marschner, US 4565693, priority 11.9.1981 to Colgate-Palmolive Co., Chem. Abstr. 99, 76683.

[23] N. Lowicki, US 4172123, priority 22.1.1970 to Grillo-Werke A.-G., Chem. Abstr. 73, 38443.

[24] R. Zekorn, Cosmetics \& Toiletries 1997, 112, 37.

[25] H. Kuhn, F. Müller, J. Peggau, R. Zekorn, J. Surfactants and Detergents 2000, 3 , 335.

[26] D. Joulain, P. Racine EP 401140, 29.5.1989 to Robertet S.A., Chem. Abstr. 114, 149323.

[27] a) K. Kulka, US 3074891, priority 24.03.1960 to Fritzsche Brothers Inc., Chem. Abstr. 59, 35761; b) T. McGee, L.K. Purzycki, V.K. Vedantam, Y.T. Tee, J. Callf, WO 2002051788, priority 22.12.2000 to Givaudan SA, Chem. Abstr. 137, 67940 .

[28] a) M. Dunkel, US 3493650, priority 13.5.1966 to Universal Oil Products Co., Chem. Abstr. 69, 69673; b) http:// www.questcosmeticactives.com/Public/ Ques7068.nsf.

[29] T. Trinh, M.T. Dodd, R.G. Bartolo, J.M. Lucas, R.Y. Buckner, M.T. Kajs, WO 9817240, priority 24.10 .1996 to Procter \& Gamble Co., Chem. Abstr. 128, 326333.

[30] E.P. Philips Jr., D.I. Richardson, 'Deodorant Ingredients' in 'Antiperspirants and Deodorants', Ed. K. Laden, C.B. Felger; Marcel Dekker Inc., New York, 1988, p 345.

[31] K. Stanzl, R. Manzo, J. Vollhardt, Dragoco Report 1998, 4, 176.

[32] A. Landa, S. Makin, 'Proceedings of the 22nd IFSCC Congress, Edinburgh, September 23-26, 2002', Edinburgh, UK, Vol. I, Chap. 5.
[33] W. Beilfuss, K.H. Diehl, H. Eggensperger, P. Oltmanns, EP 599433, priority 26.11.1992 to Schuelke und Mayr GmbH, Germany, Chem. Abstr. 120, 330813.

[34] A. Natsch, H. Gfeller, P. Gygax, J. Schmid, Int. J. Cosmetic Sci. 2005, 27, 115.

[35] G. Acuna, G. Frater, P. Gygax, EP 815833 , priority 24.6.1996 to Givaudan SA, Chem. Abstr. 128, 145174.

[36] A. Natsch, unpublished results.

[37] J.C. Powers, J.W. Harper in 'Proteinase inhibitors', Ed. Barrett and Salvesen, Elsevier, London, New York, 1986, p. 219.

[38] A. Natsch, G. Acuna, M.-C. Fournie-Zaluski, H. Gfeller, WO 02092024, priority 14.5.2001 to Givaudan SA and Pharmaleads, Chem. Abstr. 137, 380936.

[39] F. Schröder, A. Natsch, WO 2004043971, priority 14.11.2003 to Givaudan SA, Chem. Abstr. 141, 7280.

[40] K. Torii, C. Egma, EP 77047, priority 9.10.1981 to Shiraimatsu Shinyaku Co., Ltd., Chem. Abstr. 99, 10705.

[41] L.B. Buck, R. Axel, Cell 1991, 65, 175.

[42] L.B. Buck, Cell 2000, 100, 611.

[43] M. Malnic, J. Hirono, T. Sato, L.B. Buck, Cell 1999, 96, 611.

[44] a) K.J. Kessler, S.L. Sullivan, L.B. Buck, Cell 1993, 73, 597; b) R. Vassar, J. Ngai, R. Axel, Cell 1993, 74, 309.

[45] S. Zozulya, F. Echeverri, T. Nguyen, Genome Biology 2001, 2, research0018.10018.12 .

[46] E. Shirokova, K. Schmiedeberg, P. Bedner, H. Niessen, K. Willecke, J.-D. Raguse, W. Meyerhof, D. Krautwurst, J. Biol. Chem. 2005, 280, 11807.

[47] a) M. Spehr, G. Gisselmann, A. Poplawski, J.A. Riffell, C.H. Wetzel, R.K. Zimmer, H. Hatt, Science 2003, 299, 2054; b) K. Kajiya, K. Inaki, M. Tanaka, T. Haga, H. Kataoka, K. Touhara, J. Neurosci. 2001, 21, 6018.

[48] F. Sallman, A. Veithen, M. Philippeau, WO 2006094704, priority 3.3.2005 to Chemcom S.A., Chem. Abstr. 145, 306864.

[49] D.M. Stoddart, 'The Scented Ape: The Biology and Culture of Human Odor', Cambridge University Press, 1990. 